to defeat even the fastest computers.

In three dimensions the problems are even worse. As well as the need for vastly larger number of particles, there are no agreed rules of interaction. The difficulty is that there is no three-dimensional equivalent to the hexagonal lattice that produces isotropic behaviour. One possible solution is to go to a compact fourdimensional system where suitable lattices are known to exist (P. Lallemand, L'Ecole Normale Superieure; Frisch). Another is to alternate between several different lattices on successive time steps (Wolfram).

Perhaps the most convincing demonstrations of the technique are the calculations of measurable physical quantities. Researchers have validated their progams by correctly calculating quadradic velocity profiles of plane Poiseuille flow (G. Zanetti, Univ. Chicago), boundary layer profiles (Lallemand, D. d'Humieres) and vortex-shedding rates (B. Nemnich, Thinking Machines Corp.). The most dramatic demonstrations were the animated videotapes showing clear examples of vortex shedding (see cover photograph) and the propagation of shock waves. A specialpurpose parallel computer (A. Clouqueur, L'Ecole Normale Superieure) at the conference allowed participants to try such experiments in real time.

The critical test will be whether these techniques are more useful than conventional methods. This will depend on two things, accuracy and cost. It seems likely that at high Reynolds numbers these discrete simulations will not give the same answers as numerical methods. There was heated discussion at the meeting as to which answers would be correct. The possibility was even raised that such calculations may, in some cases, be more accurate than the Navier-Stokes equations themselves, because they are in some senses closer to the particulate physical reality. Such speculations were dismissed as unlikely by most of the participants. The cost question is complicated by the fact that the parallel computers that makes this possible also reduce the cost of traditional methods. Analytical techniques using renormalization methods are also being developed (S. Orszag, V. Yakhot, Princeton Univ.)

In a sense the Navier-Stokes equations are serving as a test bed for this style of discrete computation. There are already preliminary result applying these techniques to plasma flow (D. Montgomery, Dartmouth College; G. Doolen, Los Alamos) and to chemical reactions (G. Searby, Univ. Marseilles). Other potential areas of application include stress-strain analysis and the simulation of selfgravitating systems. Whether or not these new methods turn out to be superior, they are likely to offer some stimulating competition to traditional techniques.

W. Daniel Hillis is in the Thinking Machines Corporation, 245 First Street, Cambridge, Massachusetts 02142-1214, USA.

\title{
Cosmology
}

\section{A new twist for cosmic strings}

\section{Craig Hogan}

Cosmic strings are simple objects that arise in an elegant way from fundamental theory, which is perhaps why theorists have lavished so much attention on them as possible causes of galaxy formation and clustering even though there is no direct evidence for their existence. Unfortunately, if strings actually did exist in the real Universe, then one might expect a series of inelegant complex responses characteristic of many real astrophysical systems. M.J. Rees (Mon. Not. R. astr. Soc. 222, 27-32P; 1986) has recently outlined a situation in which such nonlinear responses could swamp the effects expected in a more naive theory, and even thereby generate structure on a much larger scale.

The Rees model is based on the observation that as a string sweeps through matter it leaves a 'wake' (which I described in a News and Views article, see Nature 310, 365; 1984). The matter on each side of the sheet swept out behind a cosmic string is given a kinematically induced velocity of several kilometres per second (for typical parameters) towards the sheet (see figure). For collisionless matter, this produces a wedged-shaped region (with the string at the leading edge) in which matter is streaming in two opposite directions. But for normal collisional gas, such as exists everywhere in the early Universe, counter-streaming is impossible, and instead matter crashes into a wedge-shaped shock front where it is slowed down and its energy thermalized. The heated gas then cools, so that the string leaves a thin wake of cool dense gas.

Rees points out that although these wakes comprise only a tiny fraction $\left(\sim 10^{-6}\right)$ of the matter, they can be coherent on the scale of the strings. Because strings move close to the speed of light, the coherence scale of the string network is generally the scale of the Universe itself (the 'horizon size', or the expansion time divided by the speed of light), and the effect is to produce thin cooled sheets of gas stretching across the visible Universe. Because of the cosmic expansion, this scale was smaller in the past than now, but nevertheless the first such sheets to form after the Universe

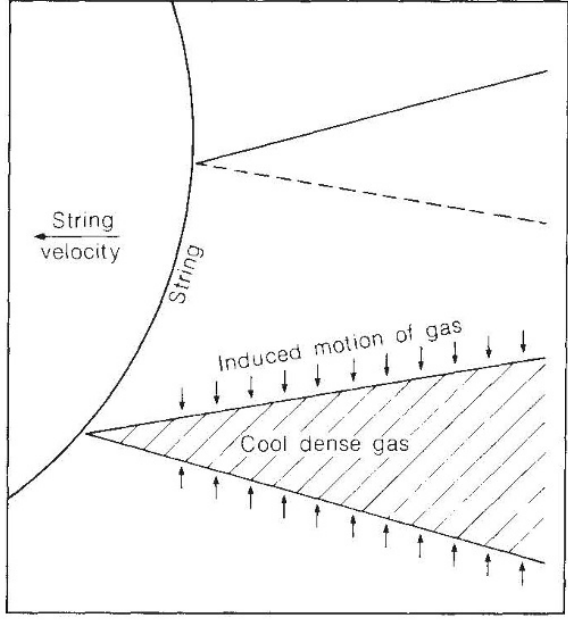

The wake produced by a cosmic string. Counter-streaming gas behind the moving string shocks and cools to form a thin dense sheet. The sheet thickness is greatly exaggerated here; in actuality the opening angle of the wedge is of the order of the ratio of gas velocity (a few $\mathrm{km} \mathrm{s}^{-1}$ ) to string velocity (a few hundred thousand $\mathrm{km} \mathrm{s}^{-1}$ ).

recombined at $10^{-3}$ its present size (redshift of $10^{3}$ ) would now have a size of the order of $100 \mathrm{Mpc}$, comparable with the scale of giant sheet-like structures sometimes claimed to be observed in the galaxy distribution and much larger than the typical, 20-Mpc clustering scale of galaxies.

String-induced sheets of cool gas are, however, too thin to make galaxies directly, and in any case the mass involved in the wakes is much too small to be directly responsible for the bulk of the observed clustering. But the sheets may be unstable, and the indirect effects of inducing local gravitational collapse could be very significant. The gas could, for example, form sheets of stars, whose luminosity, metal enrichment or explosive energy could trigger or modify the formation of galaxies; independently of the details of how this occurred, it would happen with a coherence scale of $\sim 100 \mathrm{Mpc}$. The scenario is most unique in this respect, insofar as correlations on a $100-\mathrm{Mpc}$ scale are introduced without actually moving matter this far, indeed they are introduced at a time (redshift $\sim 200$ ) when the bulk of the gas has not collapsed into structures on any scale and is still uniformly distributed.

Because so many of the pieces of the galaxy-formation puzzle remain to be fitted together, it is not yet clear whether this scheme will find a place in the final assembled picture of the origin and evolution of cosmic structures. On the other hand it is certainly an interesting new embellishment of the cosmic string model and one that is likely to be incorporated into future unified accounts of string-induced galaxy formation.

Craig Hogan is at the Steward Observatory, University of Arizona, Tucson, Arizona 85721, USA. 